\title{
Vegetación de un bosque subandino en Bolívar, Santander-Colombia
}

\author{
Nohora Alba Camargo-Espitia, Pablo Andrés Gil-Leguizamón \\ \& María Eugenia Morales-Puentes \\ Grupo Sistemática Biológica, Herbario UPTC, Escuela de Biología, Facultad de Ciencias, Universidad Pedagógica y \\ Tecnológica de Colombia. Av. Central Norte, Tunja, Colombia; nohora.camargo@uptc.edu.co, \\ pablo.gil@uptc.edu.co,maria.morales@uptc.edu.co
}

Recibido 19-III-2019. Corregido 15-VII-2019. Aceptado 30-VII-2019.

\begin{abstract}
Subandean forest vegetation in Bolívar, Santander-Colombia. We made a compositional and structural analysis in a subandean forest fragment in the municipality of Bolivar; area affected by deforestation, habitat loss and expansion of agriculture being stressors that have limited the development of these fragments. Forest is located between 1400 and $1800 \mathrm{~m}$, at $24^{\circ} \mathrm{C}$ and $2000-3000 \mathrm{~mm} /$ year. We made 10 transects $\left(100^{*} 5\right.$ $\mathrm{m}$ ), we recorded types of growth such as trees, shrubs, herbaceous, palmoid, liana, epiphyte, hemiepiphyte, parasite, tree fern and morphological data. We assessed families and genera composition using diversity, dominance, equitability and similarity indexes; IVI, IPF, IVF and frequency distribution (altitude, coverage, DAP). We recorded 102 families, 257 genera and 483 species, with 73 dicotyledons (72\%), 13 monocotyledons (13 $\%)$ and 15 pteridophytes (15\%). Families with the highest genera and species number were: Rubiaceae (15 genera/29 species), Piperacae (2/25), Gesneriaceae (11/19), Melastomataceae (8/19), Solanaceae (4/16) Araceae and Urticaeceae (7/15 each one). Genera with the highest species number were: Piper (16 species), Miconia (10), Ficus, Peperonia and Solanum (9) Anthurium and Passiflora, Siparuna, Pilea and Zanthoxylum (6). Dominance and diversity indicated species uniformity, supported in the forest equitability. The similitude between transects is $24 \%$ denoting a high species turnover. The herbaceous growth was the better represented with 56 families and 166 species. This research showed a high diversity and new records for Santander, with Passiflora engleriana Harms Endangered (EN), Aiphanes concinna H.E. Moore (Near threatened, NT), Miconia neomicrantha Judd \& Skean and Guarea kunthiana A. Juss. (Low Concern, LC).
\end{abstract}

Key words: Eastern cordillera; conservation; diversity plant; composition; structure vegetation.

Camargo-Espitia, N. A., Gil-Leguizamón, P. A., \& Morales-Puentes, M. E. (2019). Vegetación de un bosque subandino en Bolívar, Santander-Colombia. Revista de Biología Tropical, 67(4), 989-998.

Los bosques andinos oscilan entre 1000 y $3500 \mathrm{~m}$ de elevación en áreas tropicales (Rangel-Ch., 2000; Kappelle \& Brown, 2001), abarcan diferentes ecosistemas y climas con una alta diversidad y endemismos (Castaño, 2002). Por lo anterior, estos bosques son de importancia global, debido a su extraordinaria biodiversidad y regulación hídrica (Solano, 2006). Específicamente, los bosques montanos pluviales (subandinos, andinos y altoandinos) presentan una dinámica hídrica poco convencional; en ellos, la niebla y la lluvia transportadas por el viento se convierten en un aporte adicional de agua al sistema (Tobón \& Arroyave, 2007), a pesar de las intensivas y extensivas transformaciones, causadas por los asentamientos humanos (Armenteras, Gast, \& Villareal, 2003). 
Según Cuatrecasas (1989) y Rodríguez, Armenteras, Morales, \& Romero (2006), en Colombia, la franja subandina se encuentra entre 1000 a $2400 \mathrm{~m}$ en las tres cordilleras (Occidental, Central y Oriental), y se han realizado estudios geobotánicos (Cuatrecasas, 1934), fitosociológicos (Cleef, Rangel-Ch., van der Hammen, \& Jaramillo-M., 1984) y florísticos (Gentry, 1995; Giraldo-Cañas, 1995), que han generado conocimiento en la dinámica de su vegetación. Estos bosques subandinos, por sus factores bióticos y abióticos son considerados de alta importancia nacional y mundial, como ecosistemas únicos, frágiles y estratégicos (Rodríguez et al., 2006); en éstos, la agricultura ha alcanzado su máximo desarrollo; por tanto, la vegetación y áreas conservadas han desaparecido con mayor velocidad (Franco-Rosselli, Betancur, \& Fernández-Alonso, 1997).
El desconocimiento de la riqueza florística en algunas zonas subandinas es una de las problemáticas de mayor importancia. Algunos naturalistas y científicos han realizado valiosos aportes a la composición, estructura y dinámica de la vegetación nativa presente en esta región, registrados en trabajos clásicos como los de, Caldas (1966), Cleef et al. (1984), Gentry (1993a), y avances recientes como los de Reina, Medina, Ávila, Angel y Cortés (2004), Paredes y Martínez (2005), y Hernández, Rosales y Cortés (2011). Por ello, el aporte de este estudio fue generar información sobre la diversidad de un bosque subandino (100 ha, Fig. 1) entre 1400 y 1800 m, con 483 especies, 257 géneros, 102 familias, de las cuales, cuatro especies se encuentran en alguna categoría de amenaza y se amplía el rango de distribución, además de un alto recambio de especies en el mismo bosque.

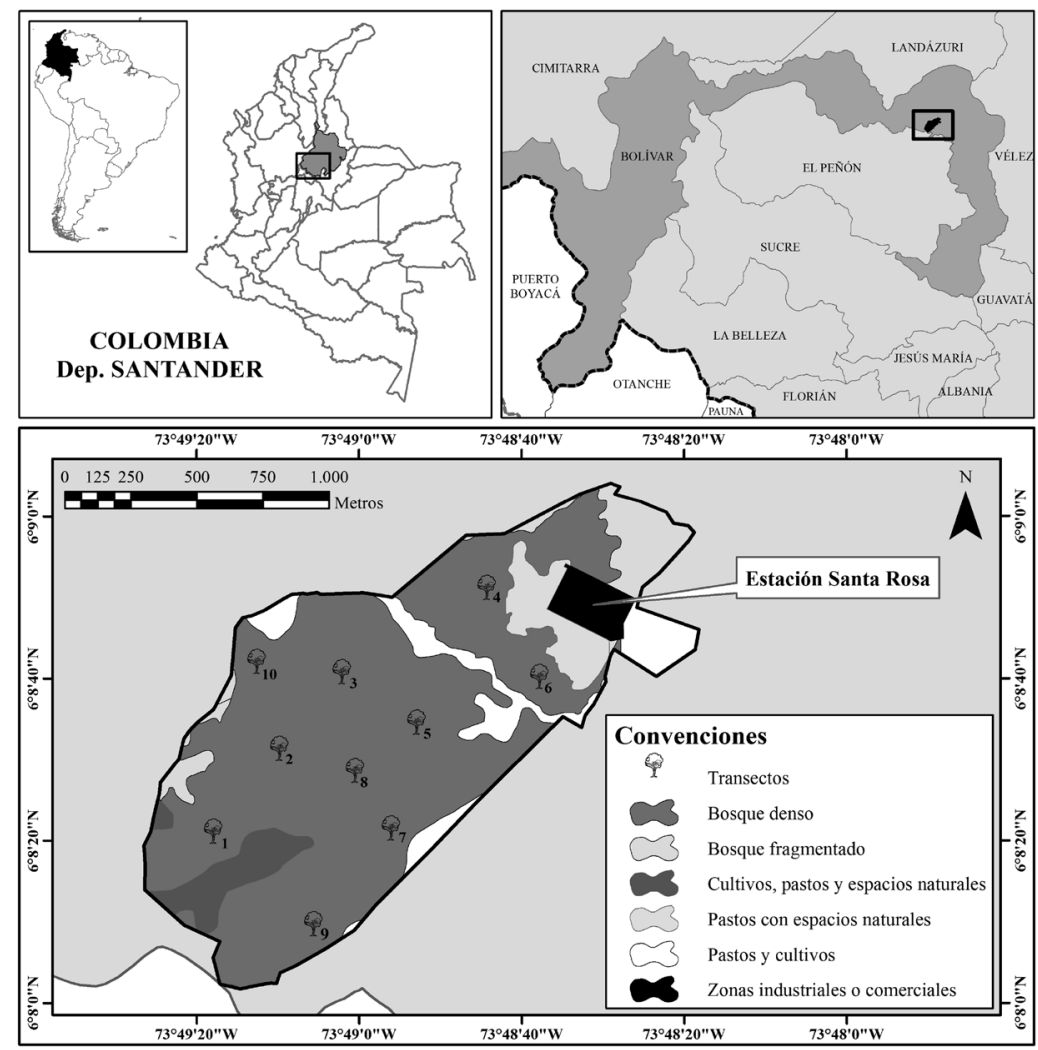

Fig. 1. Área de estudio. Transectos 1, 2, 3, 5, 8 y 10 (interior de bosque), 4, 6, 7 y 9 (borde de bosque).

Fig. 1. Study area. Transects 1, 2, 3, 5, 8 and 10 (forest interior), 4, 6, 7 and 9 (forest edge). 
Conocer estos ecosistemas, conlleva al diseño de estrategias que permitan un adecuado manejo en la conservación e importancia de estos espacios naturales para el bienestar de la población (Alvis, 2009); caso particular, el bosque subandino del municipio de Bolívar (Santander), el cual se caracteriza por un alto número de especies con coberturas boscosas amplias que ayudan a la supervivencia y conservación de hábitats para aves y mamíferos (Phillips et al., 2011).

Los estudios relacionados con flora y vegetación en los bosques subandinos generan aportes para la evaluación, manejo y conservación de estos ecosistemas (Melo-Cruz \& Vargas-Ríos, 2003). La estructura y composición de un bosque son los atributos que diferencian y caracterizan cada complejo o comunidad vegetal (Matteucci \& Colma, 1982). La estructura de un bosque está definida por su ordenamiento espacial, tanto vertical como horizontal (Kuchler, 1966; Rangel-Ch. \& Velázquez, 1997), y la composición por el conjunto de especies encontradas, en una unidad de muestreo; tales parámetros, permiten entender la dinámica y el estado de dichos ecosistemas (Whittaker, 1970).

El bosque subandino aledaño a la estación Santa Rosa comprende pequeñas áreas boscosas, con factores que actualmente causan impacto y pérdidas de la franja boscosa como son áreas de pastoreo, cultivos de lulo y mora, y entresaca selectiva (esta última actividad, es considerada una presión sobre la vegetación que allí se encuentra) (Cuta \& Gil, 2017; Gil-Novoa, Cuta-Alarcón, \& Morales-Puentes, 2017), debido a esto, este estudio buscó generar conocimiento sobre la vegetación del bosque subandino como soporte que lleve a proteger estos pocos fragmentos.

\section{MATERIALES Y MÉTODOS}

Área de estudio: en la cordillera Oriental, municipio de Bolívar (departamento de Santander), vereda Puerta de Los Cerros, en bosque de 100 ha, contiguo a la estación Santa Rosa $\left(06^{\circ} 8\right.$ N y $73^{\circ} 49$ W, Fig. 1). De acuerdo a Holdridge
(1987) es un bosque muy húmedo subtropical (bmh-ST), y según Cuatrecasas (1989), es una franja subandina, entre 1400 y $1800 \mathrm{~m}$, con precipitaciones entre 2000 a $3000 \mathrm{~mm} / \mathrm{año}$ y $18-24{ }^{\circ} \mathrm{C}$. Con afloramientos rocosos que alcanzan hasta $20 \mathrm{~m}$ de altura, y algunas plantas crecen y se adhieren a ellas, cubriéndolas casi en su totalidad (Gil-Novoa et al., 2017; Patiño-B., 2004).

Trabajo en campo: Se realizaron 10 transectos de $100 * 5 \mathrm{~m}$, de septiembre de 2014 a diciembre de 2016. Para cada individuo se registraron datos de altura $(\mathrm{m})$, circunferencia a la altura del pecho (CAP para árboles a $1.3 \mathrm{~m}$ de altura y $\mathrm{AB}$ para arbustos medido en la base de la ramificación), cobertura $\left(\mathrm{m}^{2}\right)$ y hábito o forma de crecimiento (Rangel-Ch. \& Velázquez, 1997; Font-Quer, 2001). De cada muestra se tomaron datos de aromas, exudados, colores de flores y frutos, entre otros.

Determinación taxonómica: Se llevó a cabo en el Herbario UPTC, mediante el uso de claves especializadas como, Woody Plants of Northwest South America (Gentry, 1993b) para jerarquías taxonómicas a nivel de familia y género, así como Flora Neotropica, Flora de la Guyana de Venezuela, Flora de Colombia, Flora de Mesoamérica, entre otras. Las determinaciones fueron soportadas con ejemplares depositados en el Herbario UPTC y FMB, consultas de los Herbarios virtuales NY, COL, UDBC y COAH. La corroboración fue realizada con apoyo de especialistas en algunos grupos, la clasificación se realizó bajo el sistema Angiosperm Phylogeny Group (2016), además de la citación con www.theplantlist.org, www. catalogoplantasdecolombia.unal.edu.co/es/ y www.tropicos.org.

Composición y diversidad: Se elaboró el listado de familias, géneros y especies, familias con mayor número de géneros y especies, así como géneros con mayor riqueza. La diversidad se analizó a partir de los índices de Shannon (H'), equidad y dominancia de Simpsom (D). Para comparar la similitud florística cualitativa 
entre los transectos, se calculó el coeficiente de similitud de Jaccard (Rangel-Ch. \& Velázquez, 1997), con el uso del programa estadístico PAST (Hammer et al., 2001).

Estructura: Se realizó la conversión de los valores de CAP y AB a valores de DAP (Franco-Rosselli et al., 1997; Rangel-Ch. \& Velázquez, 1997). Con datos de cobertura, altura y abundancia, se calcularon los índices de predominio fisionómico (IPF), valor de importancia para especies (IVI) y de importancia para familias (IVF), cada uno con un valor máximo de $300 \%$. Finalmente, la distribución de los individuos se hizo con un análisis por intervalos de clase (altura, cobertura y DAP).

\section{RESULTADOS}

Composición: Se recolectaron 898 muestras, de 483 especies, en 257 géneros y 102 familias; el $72 \%$ son dicotiledóneas (73), con 192 géneros y 365 especies, el $13 \%$ son monocotiledóneas (14) con 41 géneros y 73 especies, y el $15 \%$ son pteridófitos (15), con 24 géneros y 45 especies. Las familias con mayor número de géneros y especies son Rubiaceae (15 géneros/29 especies), Piperaceae (2/25), Gesneriaceae (11/19), Melastomataceae (8/19), Solanaceae (4/16), Araceae y Urticaceae (7/15); el $39 \%$ de los géneros (99) y el $45 \%$ de las especies (217) corresponden a las 13 familias más ricas. El $51 \%$ (52) de las familias tienen uno a nueve géneros, y entre dos a nueve especies (229 especies/47\%; Tabla 1); las 37 familias restantes (37\%) tienen un género y una especie.

Los géneros con mayor riqueza de especies son Piper (16 especies), Miconia (10), Ficus, Peperomia, Solanum (9), Anthurium, Clusia (7), Passiflora, Siparuna, Pilea y Zanthoxylum (6); El $23 \%$ de las especies están en los 15 géneros más ricos (111; Cuadro 1). El $29 \%$ de los géneros (74) con dos a cuatro especies (204) y el $65 \%$ restante (168) con una especie.

Composición por formas de crecimiento: Las hierbas fueron mejor representadas
TABLA 1

Familias más ricas en géneros y especies del bosque subandino contiguo a la estación Santa Rosa (BolívarSantander)

TABLE 1

Richer families in genera and species of the subandean forest in the estación Santa Rosa (Bolívar-Santander)

\begin{tabular}{|c|c|}
\hline $\begin{array}{c}\text { Familia/No. Géneros / } \\
\text { No. Especies }\end{array}$ & $\begin{array}{c}\text { Géneros representativos / } \\
\text { No. Especies }\end{array}$ \\
\hline Rubiaceae, 15 / 29 & $\begin{array}{l}\text { Hoffmannia / } 5 \\
\text { Psychotria / } 4\end{array}$ \\
\hline Piperaceae, 2 / 25 & $\begin{array}{l}\text { Piper / } 16 \\
\text { Peperomia / } 9\end{array}$ \\
\hline Gesneriaceae, 11 / 19 & $\begin{array}{l}\text { Columnea / } 4 \\
\text { Drymonia / } 4\end{array}$ \\
\hline Melastomataceae, 8 / 19 & Miconia / 10 \\
\hline Solanaceae, 4 / 16 & $\begin{array}{l}\text { Solanum / } 9 \\
\text { Cestrum / } 4\end{array}$ \\
\hline Araceae, $7 / 15$ & Anthurium / 7 \\
\hline Urticaceae, 7 / 15 & Pilea / 6 \\
\hline Fabaceae, $9 / 14$ & $\begin{array}{l}\text { Erythrina / } 3 \\
\text { Igna / } 3\end{array}$ \\
\hline Moraceae, 4 / 14 & Ficus / 9 \\
\hline Orchidaceae, $10 / 13$ & $\begin{array}{l}\text { Dichaea / } 3 \\
\text { Epidendrum / } 3\end{array}$ \\
\hline Lauraceae, 7 / 12 & Persea / 3 \\
\hline Asteraceae, 9 / 11 & Mikania / 2 \\
\hline Acanthaceae, 3 / 9 & Aphelandra / 4 \\
\hline Clusiaceae, 2 / 9 & Clusia / 6 \\
\hline Malvaceae, 9 / 9 & Hibiscus / 1 \\
\hline Salicaceae, 5 / 9 & Casearia / 5 \\
\hline Passifloraceae, 1 / 6 & Passiflora / 6 \\
\hline Siparunaceae, 1 / 6 & Siparuna / 6 \\
\hline Rutaceae, 1 / 6 & Zanthoxylum / 6 \\
\hline
\end{tabular}

en el bosque, con 56 familias y 166 especies (31.8\%), además de ser las más abundantes (2 876 individuos/ $58.3 \%$ ); en su orden, los arbustos con 41 familias, 122 especies (23\%) y 816 individuos (16.5\%), los árboles con 49 familias, 128 especie $(24.1 \%)$ y 509 individuos $(10.3 \%)$; otras formas de crecimiento como bejucos, epífitas, hemiepífitas, parásitas, palmoides y helechos arborescentes, presentan riquezas inferiores a 28 familias, 53 especies (21.1\%) y menos de 365 individuos (14.9\%).

Diversidad: De las 10 unidades muestreadas, siete tienen valores más altos en diversidad 


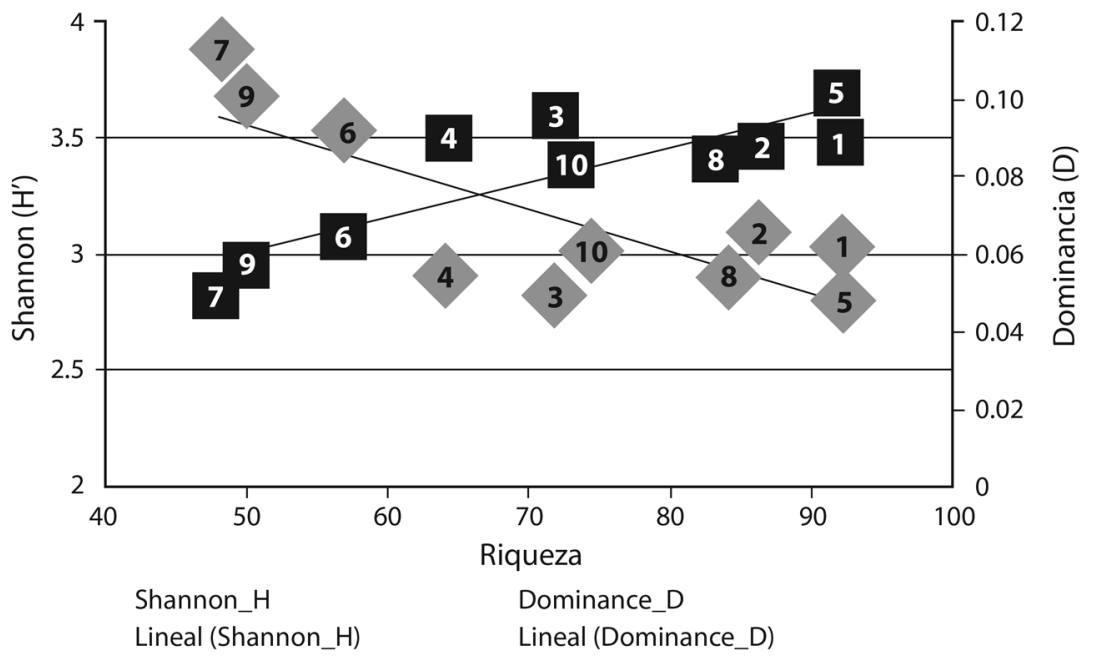

Fig. 2. Índices de dominancia y diversidad calculados para cada transecto.

Fig. 2. Dominance and diversity indexes for each transect.

y bajas dominancias (transectos del 1-5, 8 y 10 , con riquezas entre 65-92 especies). Los transectos con mayor dominancia fueron 6, 7 y $9(0.08-0.11$ D), y menor riqueza (48-57 especies; Fig. 2). Las especies más dominantes son: Triolena hirsuta (Benth.) Triana (286 individuos), Pilea sp2 (256), Chamaedorea pinnatifrons (Jacq.) Oerst. (256), Miconia sp5 (144) y Piper eriopodon (Miq.) C. DC. (84).

Similitud: Entre transectos la similitud fue $<24 \%$, lo cual indica un alto recambio de especies entre las unidades muestreadas. Los transectos 3-8 y 6-10 presentaron la mayor similitud (20\%), mientras que el transecto 4 fue el más disímil (10\%) (Fig. 3).

Estructura: Los parámetros que definen la importancia ecológica (IVI) en este bosque, son en su orden la dominancia, densidad y frecuencia. Las especies arbóreas con mayor representatividad ecológica son, Chrysochlamys dependens Planch. \& Triana (119.7\%), Critoniopsis sp1 (80.6\%), Miconia sp7 (67.5 \%), Aniba sp1 (62\%) y Miconia sp5 (59.2 \%) y las arbustivas son, Cinchona pubescens Vahl (93.9 \%), Aegiphila sp1 (43.6\%), Oreopanax macrocephalus Decne. \& Planch.

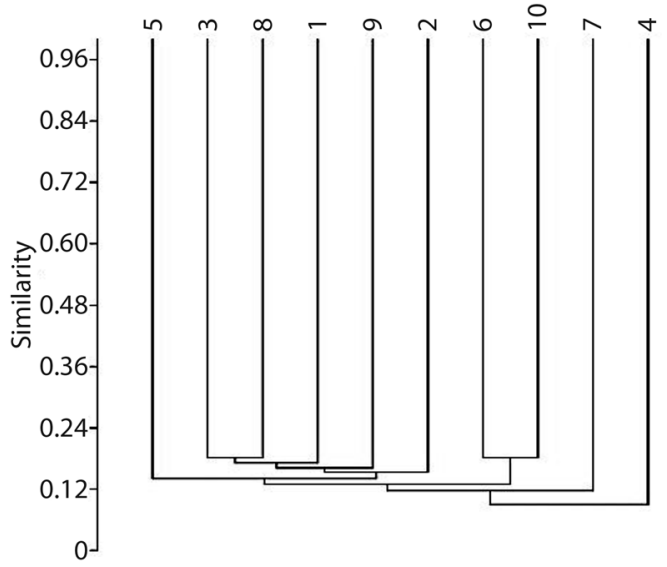

Fig. 3. Dendrograma de similitud para las 10 unidades de muestreo realizadas en el área de estudio.

Fig. 3. Dendrogram of similarity for the 10 samples unities in the study area.

ex Wedd. (52.1\%), Miconia sp5 (50.2\%) y Miconia sp7 (67.5\%).

Índice de predominio fisionómico (IPF): Los parámetros que definen la dominancia de especies en este bosque en su orden son, área basal, cobertura y densidad. Las especies arbóreas con mayor dominancia son, C. dependens (148.9\%), Critoniopsis sp1 (83.6\%), Aniba sp1 (79.8 \%) Cinnamomum sp. (71.6\%) 
y Pourouma sp. (69.2\%) y las arbustivas son: C. pubescens (108.6\%), Miconia sp7 (73.6 \%), Aegiphila sp1 (72.9\%), O. macrocephalus (58.6 \%) y Miconia sp5 (50.6\%).

Índice de valor de importancia para familias (IVIF): En su orden son, Arecaceae (365 ind/ $21.6 \%$ ), Melastomataceae (232/3.7 \%), Piperaceae (118/6.9\%) y Clusiaceae (107/6.3\%).

\section{Distribución de individuos por interva- los de clase:}

Altura: Las hierbas son las más abundantes, y tienen mayor número de individuos en el primer intervalo (intervalo 0.3-2.11 m/560 individuos $/ 33 \%$ ); entre 2.12-9.39 $\mathrm{m}$ (cuatro intervalos) son abundantes los arbustos (574 individuos $/ 34 \%$ ), árboles (379/22\%), palmoides $(47 / 2.8 \%)$ y helechos arborescentes $(3 / 0.2$ $\%$ ); en los siguientes seis intervalos $(>9.4 \mathrm{~m})$, se agrupa el $8 \%$ de la abundancia (130). Cecropia angustifolia Trécul con dos individuos es la más alta $(20 \mathrm{~m})$.

Cobertura: el $86 \%$ de los individuos (1 454) en el primer intervalo (intervalo 0.03$19.25 \mathrm{~m}^{2}$ ), seguido del $9 \%$ (146) con coberturas entre 19.26-38 $\mathrm{m}^{2}$; los restantes intervalos (9; 38.49-230.7 $\left.\mathrm{m}^{2}\right)$, corresponden al $5 \%$ de los individuos (93). Pourouma sp., presentó la mayor cobertura con $222 \mathrm{~m}^{2}$.

$D A P$ : el $78 \%$ de la abundancia $\left(\begin{array}{ll}1 & 055\end{array}\right)$ se distribuyó en el 1er. intervalo (intervalo 0.31-10.14 cm), un $21 \%$ (283) en los cinco intervalos siguientes $(10.14-59.29 \mathrm{~cm})$. Entre $59.29-98.61 \mathrm{~cm}$ (cuatro intervalos) se registraron cuatro individuos $(0.3 \%)$ y entre 98.61 $108.44 \mathrm{~cm}$ se registró a Ficus sp1.

\section{DISCUSIÓN}

Composición: Rubiaceae, Gesneriaceae, Piperaceae y Melastomataceae son las familias con mayor riqueza (29-17 especies), igualmente lo indica Reina et al. (2004) y Paredes y Martínez (2005) en el mismo rango altitudinal; a su vez, tienen amplia distribución en la cordillera Oriental (Ocaña, 2005; Medina, Herrera, Ávila, Chaparro, \& Cortés, 2010), estos grupos son diversos en los ecosistemas subandinos, proveen sustratos para la supervivencia de otros organismos y son fuente de alimento para fauna, además se encuentran en bosques secundarios o son pioneras en la sucesión (García, Suárez, \& Daza, 2010); sin embargo, algunas especies pueden tener distribución restringida (Gentry \& Emmons, 1987; Bernal, Gradstein, \& Celis, 2015).

Los géneros Piper, Ficus, Miconia, Anthurium, Clusia y Peperomia presentaron la mayor riqueza y son conocidos por su alta plasticidad ecológica, que favorece condiciones extremas o tensionantes (Gómez-Pompa, 1971; RangelCh., 1995; Giraldo-Cañas, 2001), con amplia distribución en el país desde la región subandina del Chocó, las selvas tropicales amazónicas y demás regiones del país (Gentry, 1986; Franco-Rosselli et al.,1997; Galeano, Suárez, \& Balslev, 1998).

Así también, los pteridófitos conforman el $15 \%$ de la flora del bosque, las familias más ricas son Dryopteridaceae y Polypodiaceae (9 especies cada una), como también lo fueron en los estudios de Franco-Rosselli et al. (1997), Triana y Murillo (2004), Hernández et al. (2011); tal riqueza se debe a factores climáticos (alta precipitación, humedad relativa y neblina constante), suelos con abundantes nutrientes y variadas formas de crecimiento (epífitas, hemiepífitas y terrestres), que favorecen su establecimiento (Krömer, Acebey, Kluge, \& Kessler, 2013; Acebey, Krömer, Vázquez-Torres, \& Tejero-Díez, 2015). Los géneros Elaphoglossum y Polystichum son los más ricos de la familia ( 3 especies cada uno) (Triana \& Murillo, 2004).

Forma de crecimiento: Las hierbas son las más abundantes y ricas, sin embargo, especies arbóreos se encontraron en este estrato como plántulas, por ejemplo: Micropholis guyanensis (A. DC.) Pierre (6 individuos), $C$. dependens (5), G. kunthiana y Notopleura cf. macrophylla (Ruiz \& Pav.) (4), Neea cf. divaricata Poep. \& Endl. (3), Brosimum utile (Kunth) Oken, Cupania americana L. y Oreopanax capitatus (Jacq.) Decne. \& Planch. (2), Clusia cf. eugenioides Planch. \& Linden ex Planch. 
\& Triana y Oreopanax aff. incisus (Schult.) Decne. \& Planch. (1), siendo evidencia de futuros recambios (Rangel-Ch., 1995).

Diversidad: Los valores obtenidos con los índices de dominancia y diversidad, muestran abundancias proporcionales entre especies en los 10 transectos, lo que indica que el bosque presenta una alta diversidad y baja dominancia. En pocos sectores del bosque, la dominancia de T. hirsuta, Pilea sp2, C. pinnatifrons, Miconia sp5 y $P$. aff. eriopodon, se debe a sus formas de crecimiento (herbáceas, arbustivas y palmoides), sin embargo, no son una generalidad en el área de estudio.

La diversidad fue mayor en interior de bosque (72-92 especies), que en cercanías al borde y contiguo a caminos de herradura (48-65), estos bajos valores, se debe al aumento de intensidad lumínica (mayor al $50 \%$ ), mayor exposición a la oscilación térmica (con aumento en $3{ }^{\circ} \mathrm{C}$ ), incremento de vientos, e intervención humana, lo cual ejerce cambios en la abundancia y distribución de las especies (Lezcano, Finegan, Condit, \& Delgado, 2001).

Estructura: La importancia ecológica y dominancia de las especies, soportan la heterogeneidad identificada dentro del ecosistema; $C$. dependens, Pourouma sp., Sloanea aff. brevispina Earle Sm., Aniba sp. y Cinamomum sp., presentaron los mayores valores de IVI e IPF, por ser individuos de gran tamaño, con CAP hasta de $3.5 \mathrm{~m}$, y alturas hasta $19.5 \mathrm{~m}$, tales resultados coinciden con datos registrados en otros bosques subandinos, donde la importancia ecológica se debe al dominio de especies arbóreas de fustes amplios (Dueñas, Betancur, \& Galindo, 2007).

De acuerdo a Gentry (1986), es frecuente que la alta densidad de palmas sea representativa en los bosques húmedos tropicales, como se observó en el área de estudio, con individuos hasta de $6 \mathrm{~m}$ de altura, coberturas que llegan a $15.2 \mathrm{~m}^{2}$ y CAP hasta de $0.36 \mathrm{~m}$; tales características propician sombra, que restringen la entrada de luz al sotobosque y posiblemente limitan el crecimiento de otras especies (Dueñas et al., 2007).

Intervalos de clase: La mayor cantidad de individuos están en los intervalos inferiores, también lo mencionan Cantillo y Fajardo (2004), Dueñas et al. (2007) y García et al. (2010) en estudios florísticos realizados en bosques tropicales y templados, donde a medida que aumenta el valor de los rangos de clase, el número de individuos decrece. El escaso número de individuos con diámetros superiores a $0.29 \mathrm{~m}$ (28), puede indicar intervenciones pasadas, y no todos los individuos en esta comunidad llegan a desarrollos avanzados (Lamprecht, 1990; Lezcano et al., 2001). La alta abundancia en diámetros inferiores, se relaciona con recambio de individuos, que, sumado a la alta diversidad, permiten la variabilidad ecofisiológica y de crecimiento (Araujo \& Iturre, 2006).

40 familias con un solo género, y 160 géneros con una especie, muestran la singularidad de este bosque, comparado con la "Reserva Biológica Chachalú" (con similar gradiente altitudinal a $180 \mathrm{~km}$ de distancia), la cual es área protegida, y registra una composición similar a la de Santa Rosa (443 especies; Reina et al., 2004); sin embargo, las riquezas de especies difieren hasta un $50 \%$, lo que indica un alto recambio a nivel local.

Este bosque es de interés para la conservación, ya que se registraron especies con categoría de peligro de extinción: Juglans neotropica Diels, Cedrela odorata L., P. engleriana (EN), A. concinna y Otoba cf. gracilipes (A.C. Sm) A.H. Gentry (NT) y otras 45 especies en Preocupación Menor (LC), estos datos hacen necesario adelantar estudios que permitan conocer la dinámica de este bosque en pro del mantenimiento de la diversidad. Las especies $P$. engleriana, catalogada En Peligro (EN), A. concinna (casi amenazada, NT), M. neomicrantha y $G$. kunthiana (Preocupación Menor, LC), se identificaron como nuevos registros de distribución para Santander.

Declaración de ética: los autores declaran que todos están de acuerdo con esta publicación 
y que han hecho aportes que justifican su autoría; que no hay conflicto de interés de ningún tipo; y que han cumplido con todos los requisitos y procedimientos éticos y legales pertinentes. Todas las fuentes de financiamiento se detallan plena y claramente en la sección de agradecimientos. El respectivo documento legal firmado se encuentra en los archivos de la revista.

\section{AGRADECIMIENTOS}

\section{Al Convenio 5211740 UPTC \& Ecopetrol} S.A. con el proyecto "Inventario de flora y fauna, estudio fitofenológico y disponibilidad de alimentos en un área boscosa de 100 ha contigua a la Estación Santa Rosa (Bolívar-Santander)". A la Universidad Pedagógica y Tecnológica de Colombia, al Herbario UPTC y a los integrantes del Grupo Sistemática Biológica. A los especialistas R. Callejas (Piperaceae), L. Clavijo (Gesneriaceae), M. Bonilla (Orchidaceae y Passifloraceae), C. Castro (Orchidaceae), D. Giraldo (Poaceae y Marcgraviaceae), C. Aguirre (Passifloraceae), J. Murillo (Euphorbiaceae), C. Parra (Myrtaceae), y a los generalistas R. Sánchez y D. Cárdenas.

\section{RESUMEN}

En este estudio se calculó la composición y estructura de un fragmento de bosque subandino en el municipio de Bolívar; área afectada por deforestación, pérdida de hábitat y expansión de la agricultura siendo factores que han limitado el desarrollo de estos fragmentos. Dicho bosque se encuentra entre los $1400-1800 \mathrm{~m}$ altitudinales, a $24^{\circ} \mathrm{C}$ y 2 000-3 $000 \mathrm{~mm} /$ año. Se realizaron 10 transectos $(100 \times 5 \mathrm{~m})$, en ellos se registraron las siguientes formas de crecimiento: arbórea, arbustiva, herbácea, palmoide, bejuco, epífita, hemiepífita, parásita y helecho arborescente, además de la recopilación de datos morfológicos. La composición de familias y géneros se evaluó a través de índices de diversidad, dominancia, equidad y similitud; para la estructura se calcularon IVI, IPF, IVF e intervalos de clase (altura, cobertura y DAP). Se registraron 102 familias, 257 géneros y 483 especies, con 73 dicotiledóneas (72\%), 13 monocotiledóneas $(13 \%)$ y 15 pteridófitas (15\%). Las familias con mayor número de géneros y especies fueron: Rubiaceae (15 géneros/29 especies), Piperaceae (2/25), Gesneriaceae (11/19), Melastomataceae (8/19), Solanaceae (4/16), Araceae y Urticaceae (7/15). Los géneros más ricos en especies fueron: Piper (16 especies), Miconia (10), Ficus, Peperomia y Solanum (9), Anthurium y Clusia (7) Passiflora, Siparuna, Pilea y Zanthoxylum (6). La dominancia y diversidad evidenció uniformidad en las especies, apoyado en la equitatividad del bosque. La similitud entre transectos es del $24 \%$ indicando un alto recambio de especies. El crecimiento herbáceo fue el mejor representado con 56 familias y 166 especies. Este estudio mostro una alta diversidad, así como se identifican nuevos registros para Santander, como: Passiflora engleriana Harms Endangered En Peligro (EN), Aiphanes concinna H.E. Moore (casi amenazada, NT), Miconia neomicrantha Judd \& Skean y Guarea kunthiana A. Juss. (Preocupación Menor, LC).

Palabras clave: Cordillera Oriental; conservación; diversidad de plantas; composición; estructura vegetal.

\section{REFERENCIAS}

Acebey, A., Krömer, T., Vázquez-Torres, M., \& TejeroDíez, J. (2015). Helechos y licófitos de la Reserva de la Biosfera Los Tuxtlas, Veracruz, México. Botanical Sciences, 93(2), 313-344.

Alvis, J. (2009). Análisis estructural de un bosque natural localizado en zona rural del municipio de Popayán. Revista Facultad de Ciencias Agropecuarias, 7(1), 115-122.

Angiosperm Phylogeny Group. (2016). An update of the Angiosperm Phylogeny Group classification for the orders and families of flowering plants: APG IV. Botanical Journal of the Linnean Society, 181(1), $1-20$.

Araujo, P., \& Iturre, M. (2006). Ordenación de bosques irregulares. Santiago del Estero, Argentina: Facultad de Ciencias Forestales, Universidad Nacional de Santiago del Estero.

Armenteras, D., Gast, F., \& Villareal, H. (2003). Andean forest fragmentation and the representativeness of protected natural areas in the eastern Andes, Colombia. Biological Conservation, 113(2), 245-256.

Bernal, R., Gradstein, R., \& Celis, M. (2015). Catálogo de plantas y líquenes de Colombia. Bogotá, Colombia: Instituto de Ciencias Naturales, Universidad Nacional de Colombia. Recuperado de http://catalogoplantasdecolombia.unal.edu.co/

Caldas, F. (1966). Memoria sobre la nivelación de las plantas que se cultivan en la vecindad del Ecuador. En J. Arias de Greiff, A. Bateman, A. Fernández Pérez, \& A. Soriano (Eds.), Obras completas de Francisco José de Caldas (pp. 335-344). Bogotá, Colombia: Imprenta Nacional.

Cantillo, E., \& Fajardo, A. (2004). Reserva Natural de Yotoco: Su vegetación leñosa. Colombia Forestal, 17(1), 75-93. 
Castaño, C. (2002). Colombia altoandina y la significancia ambiental del bioma páramo en el contexto de los Andes tropicales: una aproximación a los efectos futuros por el cambio climático global (Global Climatic Tensor). En C. Castaño (Ed.), Páramos y ecosistemas alto andinos de Colombia en condición de hotspots \& global climatic tensor (pp. 24-49). Bogotá, Colombia: Imprenta IDEAM.

Cleef, A. M., Rangel-Ch., J. O., van der Hammen, T., \& Jaramillo-M., R. (1984). La vegetación de las selvas del transecto Buritaca, Sierra Nevada de Santa Marta. En T. van der Hammen \& P. Ruiz-C. (Eds.), Sierra Nevada de Sta Marta transecto Alto Buritaca-La Cumbre. Estudios de Ecosistemas Tropandinos 2 (pp. 267-406). Berlin, Alemania: Cramer.

Cuatrecasas, J. (1934). Observaciones geobotánicas en Colombia. Serie Botánica, 27, 24-49.

Cuatrecasas, J. (1989). Aspectos de la vegetación natural de Colombia. Revista de la Academia Colombiana de Ciencias Exactas, Físicas y Naturales, 2(8), 147-283.

Cuta, L. E., \& Gil, J. E. (2017). Brioflora de un fragmento de bosque subandino en el municipio de Bolívar (Santander-Colombia). Ciencia en Desarrollo (Suplemento Especial), 146-147.

Dueñas, A., Betancur, A., \& Galindo, R. (2007). Estructura y composición florística de un bosque húmedo tropical del Parque Nacional Natural Catatumbo Barí, Colombia. Colombia Forestal, 10(20), 26-35.

Font-Quer, P. (2001). Diccionario de botánica. España: Ediciones Península.

Franco-Rosselli, P., Betancur, J., \& Fernández-Alonso, J. L. (1997). Diversidad florística en dos bosques subandinos del sur de Colombia. Caldasia, 19(1-2), 205-234.

Galeano, G., Suárez, S., \& Balslev, H. (1998). Vascular plant species count in a wet forest in the Chocó area on the Pacific coast of Colombia. Biodiversity and Conservation, 7(12), 1563-1575.

García, C., Suárez, C., \& Daza, M. (2010). Estructura y diversidad florística de dos bosques naturales (Buenos Aires, Dpto Cauca, Colombia). Facultad de Ciencias Agropecuarias, 8(1), 74-82.

Gentry, A. (1986). Species richness and floristic composition of Choco region plant communities. Caldasia, $15,71-91$.

Gentry, A. (1993a). Vistazo general a los bosques nublados andinos y a la flora de Carpanta. En G. Andrade (Ed.), Carpanta, selva nublada y páramo; ecología y conservación de un sistema alto andino (pp. 67-79). Bogotá, Colombia: Editorial Presencia.

Gentry, A. (1993b). A field guide to the families and genera of woody plants of northwest South America
(Colombia, Ecuador, Peru) with supplementary notes on herbaceous taxa. Washington, USA: Conservation International.

Gentry, A. (1995). Patterns of diversity and floristic composition in neotropical montane forests. En S. P. Churchill, H. Balslev, E. Forero, \& J. L. Luteyn (Eds.), Biodiversity and conservation of neotropical montane forests (pp. 103-126). New York, USA: The New York Botanical Garden.

Gentry, A., \& Emmons, L. (1987). Geographical variation in fertility, phenology and composition of the understory of Neotropical forest. Biotropica, 19(3), 216-227.

Gil-Novoa, J. E., Cuta-Alarcón, L. E., \& Morales-Puentes, M. E. (2017). Riqueza y distribución de musgos en un bosque subandino en Bolívar-Santander, Colombia. Revista Biología Tropical, 65(4): 1397-1406.

Giraldo-Cañas, D. (1995). Estructura y composición de un bosque secundario fragmentado en la cordillera Central, Colombia. En S. P. Churchill, H. Balslev, E. Forero, \& J. Luteyn (Eds.), Biodiversity and conservation of Neotropical montane forests (pp. 159-168). New York, USA: The New York Botanical Garden.

Giraldo-Cañas, D. (2001). Análisis florístico y fitogeográfico de un bosque secundario pluvial andino, cordillera Central (Antioquia, Colombia). Darwiniana, 39(3-4), 187-199.

Gómez-Pompa, A. (1971). Posible papel de la vegetación secundaria en la evolución de la flora tropical. Biotropica, 3, 125-135.

Hammer, Ø., Harper, D. A. T., \& Ryan, P. D. (2001). Past: Paleontological Statistics Software Package for Education and Data Analysis. Palaeontologia Electronica, 4(1), 9. Recuperado de http://palaeo-electronica. org/2001_1/past/issue1_01.htm

Hernández, M., Rosales, N., \& Cortés, S. (2011). Riqueza y diversidad florística de un bosque de niebla subandino en la Reserva Forestal Laguna De Pedro Palo (Tena-Cundinamarca, Colombia). Revista Facultad de Ciencias Básicas, 7(1), 32-47.

Holdridge, L. (1987). Ecología basada en zonas de vida. San José, Costa Rica: Instituto Interamericano de Cooperación para la Agricultura (IICA).

Kappelle, M., \& Brown, A. (2001). Bosques nublados del Neotrópico. Santo Domingo de Heredia, Costa Rica: Instituto Nacional de Biodiversidad (INBio).

Krömer, T., Acebey, A., Kluge, J., \& Kessler, M. (2013). Effects of altitude and climate in determining elevational plant species richness patterns: a case study from Los Tuxtlas, Mexico. Flora-Morphology, Distribution, Functional Ecology of Plants, 208(3), 197-210. 
Kuchler, A. (1966). Analyzing the physiognomy and structure of vegetation. Annals of the Association of American Geographers, 1(56), 112-127.

Lamprecht, H. (1990). Silvicultura en los trópicos. Los ecosistemas forestales en los bosques tropicales y sus especies arbóreas, posibilidades y métodos para un aprovechamiento sostenido. Alemania: Gesellschaft für Technische Zusammenarbeit.

Lezcano, H., Finegan, B., Condit, R., \& Delgado, D. (2001). Variación de las características de la comunidad vegetal en relación al efecto de borde en fragmentos de bosque Las Pavas, Cuenca del Canal de Panamá. Revista Forestal Centroamericana, 38, $33-38$

Matteucci, S., \& Colma, A. (1982). Metodología para el estudio de la vegetación. Washington, USA: Secretaría General de la Organización de los Estados Americanos.

Medina, R., Herrera, E., Ávila, F., Chaparro, O., \& Cortés, R. (2010). Catálogo preliminar de la flora vascular de los bosques subandinos de la cuchilla El Fara (Santander-Colombia). Colombia Forestal, 13(1), 55-85.

Melo-Cruz, O., \& Vargas-Ríos, R. (2003). Evaluación ecológica y silvicultural de ecosistemas boscosos. Ibagué, Colombia: Universidad Del Tolima.

Ocaña, Y. (2005). Caracterización florística y estructura de unidades de bosque andino en las veredas Minas y Patios Altos del municipio de Encino Santander. Colombia Forestal, 9(18), 70-86.

Paredes, A., \& Martínez, A. (2005). Diversidad y caracterización florística y estructural de la vegetación secundaria de la Reserva Biológica Cachalú, con propósitos de restauración (Encino-Santander) (Tesis de Pregrado). Universidad Distrital Francisco José de Caldas, Colombia.

Patiño-B., G. (2004). Esquema de ordenamiento territorial (EOT) municipio de Bolivar-Santander, documento técnico-etapa preliminar. Santander, Colombia: Universidad Industrial de Santander.

Phillips, J., Duque, A., Yepes, A., Cabrera, K., García, M., Navarrete, P., \& Cárdenas, D. (2011). Estimación de las reservas potenciales de carbono almacenadas en la biomasa aérea en bosques naturales de Colombia. Estratificación, alometría y métodos analíticos.
Bogotá, Colombia: Instituto de Hidrología, Meteorología y Estudios Ambientales (IDEAM).

Rangel-Ch., J. (1995). La diversidad florística en el espacio andino de Colombia. En S. Churchill, H. Balslev, E. Forero, \& J. Luteyn (Eds.), Biodiversity and Conservation of Neotropical Montane Forest (pp. 187-205). New York, USA: New York Botanical Garden.

Rangel-Ch., J. (2000). Colombia diversidad biótica III, la región de vida paramuna. Bogotá, Colombia: Unibiblos Universidad Nacional de Colombia.

Rangel-Ch., J. \& Velázquez, A. (1997). Métodos de estudio de la vegetación. En J. Rangel-Ch, P. Lowy-C., \& M. Aguilar-P. (Eds.), Diversidad Biótica II. Tipos de Vegetación en Colombia (pp. 59-87). Bogotá, Colombia: Universidad Nacional de Colombia.

Reina, M., Medina, R., Ávila, F., Angel, S., \& Cortés, R. (2004). Catálogo preliminar de la flora vascular de los bosques subandinos de la Reserva Biológica Cachalú, Santander (Colombia). Colombia Forestal, 13(1), 27-54

Rodríguez, N., Armenteras, D., Morales, M., \& Romero, M. (2006). Ecosistemas de los Andes colombianos. Bogotá, Colombia: Instituto de Investigación de Recursos Biológicos Alexander von Humboldt.

Solano, C. (2006). Reserva Biológica Cachalú: 10 años de investigación en bosques de roble. En C. Solano \& N. Vargas (Eds.), Memorias del I Simposio Internacional de Roble y Ecosistemas Asociados (pp. 11-23). Bogotá, Colombia: Fundación Natural-Pontificia Universidad Javeriana.

Tobón, C., \& Arroyave, F. (2007). Inputs by fog and horizontal precipitation to the paramo ecosystems and their contribution to the water balance. En A. Biggs \& P. Cereceda (Eds.), Proceedings Fourth International Conference on Frog Collection and Dew (pp. 233-236). La Serena, Chile: Conference on Fog, Fog Collection and Dew.

Triana, L., \& Murillo, J. (2004). Los pteridófitos de un bosque subandino en el municipio de Albán, Cundinamarca (Colombia). Acta Biológica Colombiana, 9(2), 112-124.

Whittaker, R. (1970). Communities and ecosystems. Londres, Inglaterra: MacMillan Publishing. 